\title{
Tratamento de uma Coorte de Pacientes com Infarto Agudo do Miocárdio com Supradesnivelamento do Segmento ST
}

\author{
Treatment of a Cohort of Patients with Acute Myocardial Infarction and ST-segment Elevation
}

\begin{abstract}
Jamil da Silva Soares ${ }^{1}$, Nelson Robson Mendes de Souza², Jair Nogueira Filho ${ }^{3}$, Cristiane C. Cunha , Georgina Severo Ribeiro ${ }^{4}$, Ronald Souza Peixoto ${ }^{1}$, Carlos Eduardo C. Soares ${ }^{1}$, Leandro C. Soares ${ }^{5}$, Aldo Franklyn Reis ${ }^{1}$, Carlos Augusto Cardozo de Faria ${ }^{4}$

Hospital Escola Álvaro Alvim - Faculdade de Medicina de Campos, Campos dos Goytacazes, Rio de Janeiro, Rli'; Hospital Geral de Bonsucesso, Rio de

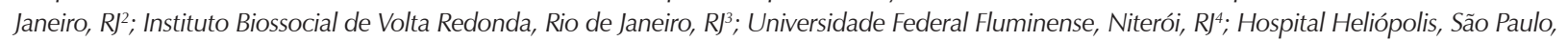
$S P^{5}$ - Brasil
\end{abstract}

\section{Resumo}

Fundamento: Trombólise e angioplastia transluminal coronariana (ATC) primária são técnicas bem estabelecidas, mas grande parte dos pacientes com infarto agudo do miocárdio com supradesnivelamento do segmento ST (IAM com SST) não as recebem quando do atendimento hospitalar.

Objetivo: Descrever tratamentos inicial e final e desfechos de uma coorte com IAM com SST.

Métodos: Analisados, da internação até a alta, 158 pacientes com IAM com SST, de uma população total de 351 pacientes internados com (SCA) nos hospitais de Campos dos Goytacazes, entre 2004 e 2006.

Resultados: Dos 158 pacientes com IAM com SST, 67,7\% chegaram ao hospital nos primeiros 180 minutos, 81,3\% em 360 minutos e 8,4\% após doze horas. Realizados 148 estudos cinecoronariográficos (93,7\%). Observadas lesões de mais de $70 \%$ em 266 territórios arteriais. Tratamento inicial foi ATC em 41(26\%), trombolíticos em 50 (32\%), com 80\% de sucesso. Tratamento clínico em 67 (42\%). Cerca de 35\% dos pacientes deveriam ser trombolizados mas não o foram. No tratamento final foram 93 ATCs, 89 delas com sucesso angiográfico $(95,7)$, sangramento $2(2,2)$, oclusão subaguda 2 (2,2\%), dissecção tronco $1(1,1)$, pseudo aneurisma $1(1,1)$. Nenhum óbito durante angioplastia; na evolução, houve dois óbitos $(2,1 \%)$. Doze pacientes submetidos a cirurgia de revascularização miocárdica (CRM). Tratamento clínico 53 (33\%), com 11 óbitos (20,7\%). Letalidade global 9,5\%, consideradas as três formas de tratamento.

Conclusão: Pacientes atendidos em tempo adequado para reperfusão, porém 1/3 deles não recebeu o procedimento. Tratamento predominante foi ATC, com baixa morbidade. Dois óbitos na evolução. Baixa letalidade global. (Arq Bras Cardiol 2009;92(6):464-471)

Palavras-chave: Infarto do miocárdio/mortalidade, terapia trombolítica, perfil de saúde, medicina baseada em evidências, letalidade. Summary
Background: Although thrombolysis and primary CTA are well-established procedures, they are not administered in a large proportion of the
patients with STEMI who arrive to the emergency rooms.

Objective: Describe initial and final the results in a cohort of STEMI patients

Methods: The study included, from hospital admission to the discharge, 158 patients diagnosed with STEMI, from a total of 351 patients with ACS admitted to hospitals in Campos dos Goytacazes, RJ, Brazil, between 2004 and 2006.

Results: Of the 158 patients with STEMI, 67.7\% arrived to the hospital within 180 minutes, $81.3 \%$ within 360 minutes, and $8.4 \%$ after twelve hours from the symptoms. Cinecoronariographic studies (148) were performed (93,7\%). Lesions of over 70\% were observed in 266 artery territories. The initial treatment was CTA in 41 (26\%), thrombolytics in 50 (32\%), 80\% of success. Clinical treatment in 67 (42\%). Approximately $35 \%$ of the patients should have undergone thrombolysis, but they didn't. During the final treatment, 93 CTAs were performed: 89 with angiographic success (95.7\%), bleeding $2(2.2 \%)$, subacute occlusion $2(2.2 \%)$, trunk dissection 1 (1.1\%), pseudoaneurism 1 (1.1\%). No deaths during angioplasty; during evolution, there were two deaths (2.1\%). Twelve patients underwent myocardial revascularization surgery (MRS), while 53 underwent clinical treatment, with 11 deaths (20.7\%). Global lethality was 9.5\%, considering the three types of treatment.

Conclusion: Patients were suitable for reperfusion, but one third of them did not have the procedure. Two deaths during evolution. The most predominant treatment was CTA, with low morbidity. Low global lethality. (Arq Bras Cardiol 2009;92(6):430-436)

Key words: Myocardial infarction/mortality; thrombolytic therapy; health profile; evidence - based medicine; lethality.

Full texts in English - http://www.arquivosonline.com.br

Correspondência: Jamil da Silva Soares •

Rua Barão de Lagoa Dourada, 409 - Centro - 28035-211 - Campos dos Goytacazes, RJ - Brasil

E-mail: jamilsoares@uol.com.br

Artigo recebido em 30/08/2007; revisado recebido em 19/03/2008; aceito em 19/03/2008 


\section{Introdução}

As doenças cardiovasculares (DCV) constituem importante causa de mortalidade em todo o mundo ${ }^{1}$, e a projeção para o ano 2020 é de que $40 \%$ dos óbitos estarão relacionados com as doenças cardiovasculares. Nesse contexto, o infarto agudo do miocárdio será a principal causa isolada ${ }^{2}$. Nos Estados Unidos, a síndrome coronariana aguda afeta 1,3 milhão de pessoas, sendo $25 \%$ homens e $38 \%$ mulheres que morrem todo ano em decorrência de $\mathrm{IAM}^{3}$. No caso de pacientes com IAM com SST, a mortalidade permanece alta, apesar dos muitos avanços terapêuticos ${ }^{4}$.

Apesar dessas constatações, o conhecimento sobre a prevalência dos fatores de risco nas diversas populações é ainda pequeno, e quase sempre nos baseamos em dados fornecidos por trabalhos realizados em populações totalmente diferentes das nossas para traçarmos políticas de controle das doenças cardiovasculares. No Brasil, existem pouquíssimos trabalhos com levantamento das características dos pacientes e práticas hospitalares, menos ainda dos seus resultados. Entretanto, visto serem as SCA a principal causa de morte e uma das mais importantes razões de internação hospitalar, tornam-se fundamentais para a saúde pública informações sobre quem se interna (idade, sexo e dados socioeconômicos), bem como sobre as formas de tratamento, evolução hospitalar e desfechos, entre outras ocorrências, relacionados a esses pacientes.

No município de Campos dos Goytacazes, Rio de Janeiro, por exemplo, onde se realizou o presente estudo, ainda não há dados disponíveis nessa área, que serão agora oferecidos por esta pesquisa. De fato, verifica-se um quase total desconhecimento desse perfil na população local, e a determinação de tais fatores poderá auxiliar na aplicação de medidas terapêuticas mais individualizadas e eficazes, acarretando melhora do prognóstico e possibilidade de orientar os gestores de saúde na criação de políticas de saúde mais adequadas para atender essa população específica de pacientes.

O somatório apresentado foi o que nos motivou a esta investigação em nossa cidade, com o objetivo de traçar o perfil epidemiológico de uma coorte de pacientes com diagnóstico de IAM com SST na alta hospitalar, visando identificar, dentro de um determinado conjunto de fatores de risco, elementos capazes de discriminar quais, dentre eles, encontram-se sob um risco maior de evolução desfavorável.

\section{Métodos}

Um estudo de coorte, observacional, prospectivo e analítico, envolvendo conhecimentos na área de epidemiologia clínica, biologia molecular e genética, foi realizado no município de Campos dos Goytacazes, situado na região norte do Estado do Rio de Janeiro. Dados demográficos em 2005 registravam uma população local de 406.985 pessoas (sexo masculino, 196.711; feminino, 210.274), com acentuada concentração urbana (364.177 pessoas), comparada à da zona rural (42.808 pessoas), e constituída de 112.037 famílias, das quais 32.258 $(28,8 \%)$ viviam com uma renda mensal de até um salário mínimo, enquanto outras 84.759 (75,6\%) tinham uma renda mensal de até cinco salários mínimos. Na área da saúde, a rede ambulatorial e hospitalar municipal dispunha de 82 unidades básicas - postos de saúde, centro de saúde e policlínicas - , distribuídas pela cidade e pelos quatorze distritos, enquanto a rede hospitalar disponibilizava 1.682 leitos, distribuídos pelos seus dez hospitais, sendo dois deles públicos, três filantrópicos e cinco da rede particular. Dessas instituições, três possuíam estrutura montada para diagnóstico de DCV, com serviços de hemodinâmica, cardiologia intervencionista e cirurgia cardiovascular, número este reduzido para dois na fase final da pesquisa. Somente um dos hospitais dispunha dos recursos de medicina nuclear. De acordo com os dados da Secretaria Estadual de Saúde, em 2005 foram constatados 1.898 óbitos por diversas causas em homens e 1.430 em mulheres, e o número de óbitos por doenças cardiovasculares em homens foi de 430 (22,65\%) e em mulheres de 428 (29,93\%).

De início, houve a participação de todos os dez hospitais da cidade: Hospital Escola Álvaro Alvim (AA), Hospital da Beneficência Portuguesa de Campos (BP), Hospital Ferreira Machado (FM), Hospital dos Plantadores de Cana (HP), Hospital Prontocárdio (PC), Hospital Geral Dr. Beda (DB), Clínica Centrocor (CC), Hospital Geral de Guarus (HG), Hospital Pró-Clínicas (PL) e Hospital da Santa Casa de Misericórdia de Campos (SC). Após doze meses, por razões diversas, os pacientes passaram a ser incluídos exclusivamente no Hospital Escola Álvaro Alvim, que se tornou o hospital-sede da pesquisa.

Foram incluídos, consecutivamente, cerca de 500 pacientes com diagnóstico de síndrome coronariana aguda, nas suas diversas formas de apresentação, constituindo, assim, um registro dessa patologia na cidade de Campos. Para efeito, porém, de apresentação deste artigo, utilizaram-se informações somente da população geral de 351 pacientes com SCA e dados específicos dos 158 pacientes com IAM com SST.

O estudo, de curto prazo, realizou-se no período entre agosto de 2004 e dezembro de 2006, com avaliação durante a fase de internação nos hospitais participantes da pesquisa.

- Critérios de inclusão: pacientes maiores de 20 anos de idade, de ambos os sexos, com síndrome coronariana aguda, com ou sem supradesnivelamento do ST; pacientes com primeiro evento coronariano agudo dentro de 72 horas do início dos sintomas ou com doença arterial coronariana (DAC) prévia.

- Critérios de exclusão: doenças neoplásicas em fase terminal, politraumatismos, demência, infarto pós ou peroperatório (cirurgias que não fossem de revascularização miocárdica).

- Diagnóstico de infarto agudo do miocárdio com supradesnivelamento do ST: foi considerado quando os pacientes apresentavam alteração dos marcadores de necrose miocárdica: aumento, seguido de gradual diminuição, de CK-MB ou CK total, excedendo duas vezes o valor superior da normalidade em pelo menos uma medida dentro de 48 horas do início do quadro agudo, ou excedendo três vezes o valor superior de normalidade em pacientes submetidos a angioplastia nas últimas 48 horas, associado a alterações eletrocardiográficas compatíveis com injúria subepicárdica nova (ou presumivelmente nova), elevação de segmento ST no 
ponto J, em duas ou mais derivações contíguas $\geq 0,2 \mathrm{mV}$ em V1, V2 e V3 e $\geq 0,1 \mathrm{mV}$ em outras derivações - ou bloqueio agudo, ou presumivelmente agudo, do ramo esquerdo.

No início da pesquisa, em cada hospital havia um médico responsável pela inclusão dos pacientes e que era acionado toda vez que ocorria uma internação por diagnóstico de SCA. $\mathrm{O}$ atendimento era feito pelas equipes de plantão das unidades de emergência dos hospitais, priorizando o diagnóstico e o controle clínico dos pacientes. Logo que possível, o respectivo médico-pesquisador entrava em contato com o paciente e seus familiares, obtendo o termo de consentimento livre e esclarecido, e, em seguida, eram colhidos os dados iniciais do paciente e realizada a coleta de amostras de sangue, incluindo também amostras sanguíneas para extração e armazenamento do DNA para futuras análises de polimorfismos genéticos relacionados com as doenças cardiovasculares.

Os pacientes incluídos foram acompanhados no que se refere à sua evolução clínica e às terapêuticas clínicas e cirúrgicas adotadas durante todo o período de internação hospitalar, inclusive as complicações e os desfechos clínicos até a alta ou o óbito. Essa limitação do tempo de acompanhamento da população-amostra, ou seja, entre a internação e a alta hospitalar, inviabilizou a avaliação dos procedimentos e desfechos em médio e longo prazos.

Não houve interferência ou controle dos pesquisadores deste estudo sobre o tipo de tratamento prescrito para cada paciente, nem em relação às rotinas estabelecidas pelos diversos hospitais participantes, exceto no que se referia a algumas medidas e dosagens previamente estipuladas na pesquisa e acordadas em reuniões com os responsáveis pelos hospitais. Assim sendo, as drogas e os procedimentos utilizados nesses locais foram os rotineiros, determinados pelos médicos assistentes, não tendo feito parte da pesquisa a utilização de medicações ou técnicas experimentais.

\section{Estatística}

Utilizou-se o programa STATA, na versão 9.1, para a montagem do banco de dados e a análise estatística.

O teste $t$ de Student foi utilizado na seguinte condição: média de duas variáveis dependentes, se a variável contínua tivesse distribuição normal ou maior que 50 observações.

Se a variável dependente fosse não-normal e os tamanhos amostrais fossem pequenos (menor que 50 observações), eram aplicados os testes de Wilcoxon, quando a variável independente apresentasse duas categorias, ou KrusKal-Wallis, quando a variável independente apresentasse mais de duas categorias. Nos casos de variável dependente nominal e variável independente categórica, era usado teste qui-quadrado, para verificar a associação entre duas variáveis categóricas. Se pelo menos $80 \%$ das frequências esperadas excedessem cinco e todas as frequências esperadas excedessem um, usava-se o teste de Fisher: se as frequências esperadas fossem menores que cinco, e o número de observações, menor que trinta. Se fosse variável independente ordinal, utilizava-se teste de Wilcoxon, como descrito anteriormente.

As fichas utilizadas para a anotação dos dados do paciente, dos resultados dos seus exames de sangue e de sua evolução intra-hospitalar encontram-se anexadas ao projeto de pesquisa original.

A presente pesquisa, resultante de uma parceria com a Universidade Federal Fluminense, foi aprovada pelo Comitê de Ética em Pesquisa da Faculdade de Medicina de Campos/Hospital Escola Álvaro Alvim, da Fundação Benedito Pereira Nunes.

O Termo de Consentimento Informado, Livre e Esclarecido foi assinado por todos os pacientes, após leitura, conforme orientações de procedimentos éticos para pesquisas genéticas, com ênfase nos consentimentos populacional e individual, publicados em 2000 pela Massachusetts Medical Society, baseados na experiência islandesa com a de Code genetics na criação do Banco de Dados de Assistência Médica Islandesa (IHD).

\section{Resultados}

Dos 158 pacientes com IAM com SST, as idades variavam de 22 a 89 anos, com média de 60,8 anos. Da amostra, 65,8\% eram do sexo masculino, com baixo nível de escolaridade, $54,4 \%$ tinham menos de quatro anos de frequência escolar e apenas $10,1 \%$ apreentavam o curso superior. A maioria dos pacientes era da religião católica $(76,5 \%)$, dependente do Sistema Único de Saúde (72,8\%) e com renda familiar de até cinco salários mínimos (65,4\%), com somente 14,8\% deles ganhando mais do que dez salários mínimos.

\section{Fatores de risco cardiovascular}

Houve alta prevalência dos principais fatores de risco, exceto do tabagismo. O diabete esteve presente em 24\% dos pacientes, hipercolesterolemia em 35\%, história familiar de coronariopatia em 52,7\%, doença arterial coronariana prévia em 15,4\% e tabagismo em 23,4\% (tab.1).

O tempo entre o início do quadro clínico e a chegada ao hospital foi menor do que três horas em $67,7 \%$ dos pacientes, e apenas 8,4\% chegaram ao pronto-atendimento após decorridas doze horas dos primeiros sintomas. Quanto à classificação de Killip, 78,7\% encontravam-se no grau I ao darem entrada no hospital (tab.1).

\section{Estudo cinecoronariográfico}

Esse estudo foi realizado em 148 pacientes (93,7\%), em algum momento durante a internação. Observaram-se lesões superiores a 70\% que envolviam 264 territórios arteriais, predominando o da artéria descendente anterior, que foi a mais acometida, tanto de forma isolada quanto associada com outras artérias. Foi essa artéria também a maior responsável pelos quadros de IAM $(57,4 \%)$, seguida pelas artérias coronária direita $(33,8 \%)$ e circunflexa $(7,5 \%)$. Observaramse duas lesões de tronco $(1,4 \%)$, sem responsabilidade pelo quadro agudo, e em dois pacientes $(1,4 \%)$ não determinamos a artéria responsável pelo referido quadro. No momento do estudo cinecoronariográfico, foi observado fluxo arterial coronariano na artéria culpada: TIMI 0 em 55 pacientes (59,1\%) e TIMI III em 30 pacientes (32,6\%). Em 107 pacientes (70,5\%), a fração de ejeção do ventrículo esquerdo, medida pela ventriculografia realizada durante a coronariografia ou, na ausência desta, pela ecocardiogafia, era superior a 55\% 
Tabela 1 - Dados básicos, principais fatores de risco, tempo para chegada ao hospital e Killip da chegada dos pacientes com IAM com SST na cidade de Campos dos Goytacazes, RJ, 2004-2006

\begin{tabular}{|c|c|}
\hline $\mathrm{n}$ & 158 \\
\hline \multirow{3}{*}{ Idade } & Média de 60,8 anos (22-89) \\
\hline & $19,7 \%<50$ anos \\
\hline & $27,9 \%>70$ anos \\
\hline Sexo & Masculino $65,4 \%$ \\
\hline \multirow{2}{*}{ Nivel de escolaridade } & Até 4 anos $(54,4 \%)$ \\
\hline & Nivel superior $(10,1 \%)$ \\
\hline Religião & 76,5 católicos \\
\hline \multirow{2}{*}{ Renda familiar (salários mínimos) } & Até 5 salários $(65,4 \%)$ \\
\hline & > 10 salários $(14,8 \%)$ \\
\hline Seguro saúde & $72,8 \%$ (SUS) \\
\hline Pessoas na família & 3,3 (média) \\
\hline Tabagismo & $37 / 159(23,4 \%)$ \\
\hline HAS (\%) & $91 / 151(60,3 \%)$ \\
\hline Diabete (\%) & $37 / 154(24 \%)$ \\
\hline Hipercolesterolemia (\%) & $42 / 120(35 \%)$ \\
\hline DAC familiar (\%) & $69 / 131(52,7 \%)$ \\
\hline DAC prévia (\%) & $24 / 156(15,4 \%)$ \\
\hline Uso de álcool & $59 / 156(27,8 \%)$ \\
\hline \multirow{2}{*}{ Tempo de chegada } & Até 3 horas $(67,7 \%)$ \\
\hline & $>12$ horas $(8,4 \%)$ \\
\hline \multirow{2}{*}{ Killip da chegada } & Killip I (78,2\%) \\
\hline & Killip II, III, IV $(21,8 \%)$ \\
\hline
\end{tabular}

HAS - hipertensão arterial sistêmica; DAC - doença arterial coronariana.

(tab. 2).

Uso de medicamentos

Observou-se uma baixa utilização dos principais medicamentos na população estudada, tanto na chegada ao hospital quanto na alta hospitalar. Na chegada, a aspirina, como medicação básica para o tratamento desses pacientes, vinha sendo utilizada em apenas $26,2 \%$ deles, os betabloqueadores em 14\%, as estatinas em 9\% e os inibidores da ECA em 29,9\%, enquanto o uso combinado de aspirina, betabloqueadores e estatina foi registrado em apenas 4,5\% dos pacientes. Na alta, a aspirina foi prescrita para $96,8 \%$ dos pacientes, os betabloqueadores para $65,8 \%$, as estatinas para $86,1 \%$, os inibidores da ECA para $76 \%$, e a associação de aspirina, betabloqueadores e estatina para apenas 57\% deles (fig. 1).

O tratamento inicial (fig. 2) realizado em 50 pacientes (32\%) foi a trombólise venosa, e dez deles (20\%), sem critérios de recanalização, foram encaminhados para angioplastia de resgate. O tempo entre a chegada ao hospital e o início da trombólise venosa situou-se entre 15 minutos e 360 minutos, com uma média de 89 minutos, e 53,1\% dos pacientes iniciaram o trombolítico dentro dos primeiros 60 minutos.
Tabela 2 - Dados angiográficos e fração de ejeção do VE dos pacientes com IAM com SST na cidade de Campos dos Goytacazes, RJ, 2004-2006

\begin{tabular}{|c|c|}
\hline Número de coronariografia & $148-93,7 \%$ \\
\hline \multirow{5}{*}{$\begin{array}{l}\text { Número de territórios com } \\
\text { lesões }>70 \%\end{array}$} & 264 \\
\hline & DA $15(77,7 \%)$ \\
\hline & CD $81(54,7 \%)$ \\
\hline & Cx $66(54,7 \%)$ \\
\hline & TCE $2(1,4 \%)$ \\
\hline \multirow{5}{*}{ Número de lesões/pac. } & Uniarterial $70,0(47,3 \%)$ \\
\hline & Biarterial $35,0(23,7 \%)$ \\
\hline & Triarterial $39,0(26,4 \%)$ \\
\hline & TCE $2,0(1,4 \%)$ \\
\hline & Sem lesões $2,0(1,4 \%)$ \\
\hline \multirow{4}{*}{ Artéria culpada } & DA $85(57,4 \%)$ \\
\hline & CD $50(33,8 \%)$ \\
\hline & $\mathrm{C} \times 11(7,5 \%)$ \\
\hline & Não ident. $2(1,4 \%)$ \\
\hline \multirow{4}{*}{$\begin{array}{l}\text { Fluxo na artéria culpada da ATC } \\
\text { (93 pacientes) }\end{array}$} & TIMI $055(59,1 \%)$ \\
\hline & TIMI I 6 (6,4\%) \\
\hline & TIMI II 2 (2,1\%) \\
\hline & TIMI III $30(32,6 \%)$ \\
\hline \multirow{4}{*}{ FEVE } & Acima de 55\% 107 (70,5\%) \\
\hline & $46 \%$ a $55 \% 30(19,7 \%)$ \\
\hline & $30 \%$ a $45 \% 11(7,2 \%)$ \\
\hline & Abaixo de $30 \% 4(2,6 \%)$ \\
\hline
\end{tabular}

$D A$ - artéria descendente anterior; $C D$ - artéria coronária direita; $C X$ - artéria circunflexa; TCE - tronco da artéria coronária esquerda; FEVE - fração de ejeção do ventrículo esquerdo.

Submeteram-se à angioplastia primária 41 pacientes (26\%), e em cerca de $40 \%$ deles os procedimentos foram realizados com tempo porta-balão menor que 90 minutos. Desses 41 pacientes, quatro evoluíram com dor precordial e sinais de isquemia, tendo sido submetidos a angioplastia após doze horas. A maioria dos pacientes (42\%) foi mantida em tratamento clínico, apesar de eles terem chegado ao prontoatendimento em tempo hábil para realizar algum tipo de reperfusão. Não houve nenhum tratamento cirúrgico como medida inicial.

Quanto ao tratamento definitivo, durante a fase hospitalar, 42 pacientes foram submetidos a angioplastia coronariana mais tardiamente para tratamento de lesões residuais ou de outras artérias não responsáveis pelo quadro agudo, embora de grande importância funcional. Indicaram-se cirurgias de revascularização miocárdica (CRM) em 18 pacientes, das quais doze foram realizadas, tendo sido revascularizadas 31 artérias (2,6 artérias por paciente), com ocorrência de dois óbitos no pós-operatório imediato, um deles por acidente vascular 


\section{Artigo Original}

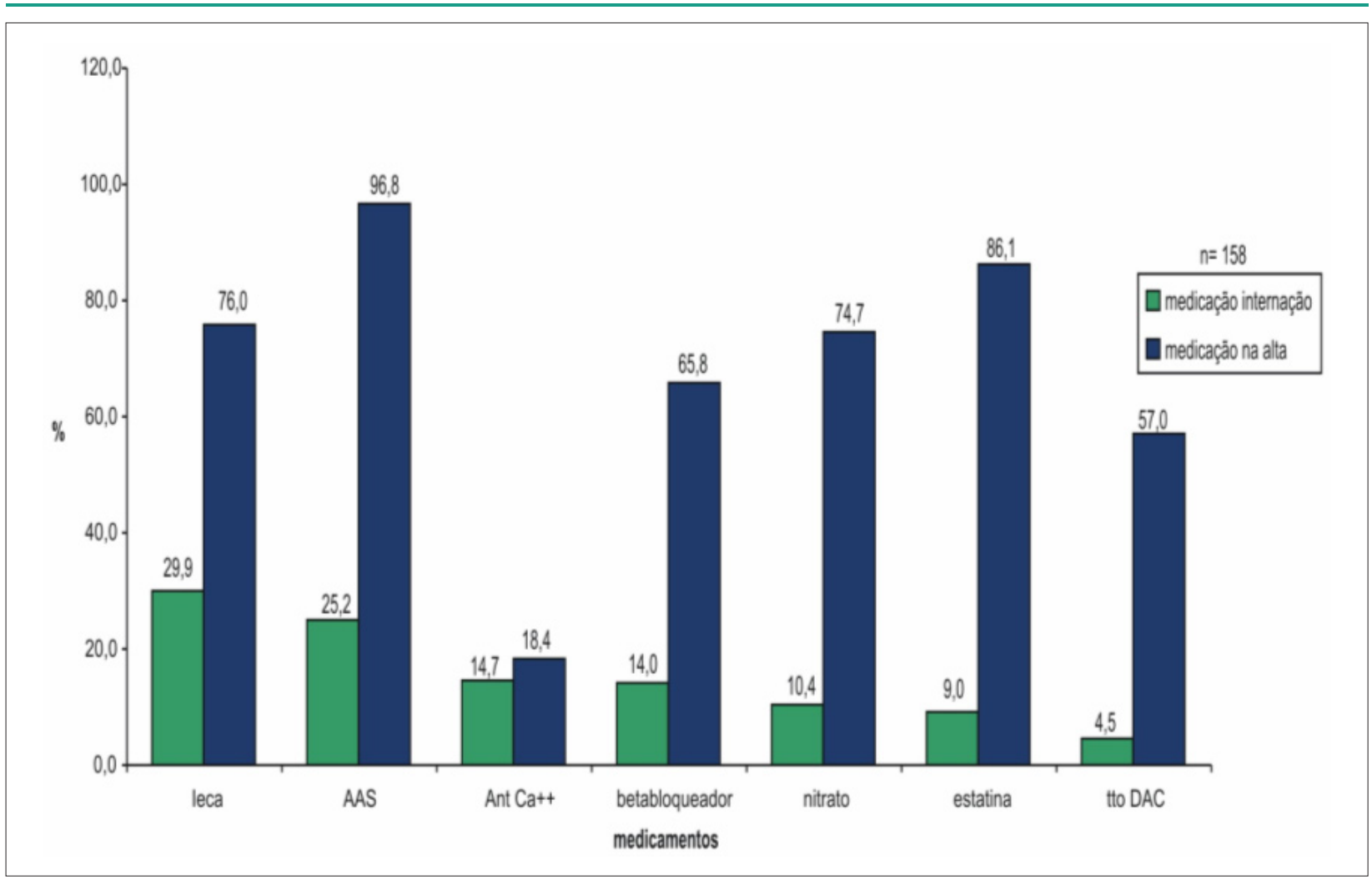

Fig. 1 - Medicações na internação e na alta hospitalar dos pacientes com IAM com SST na cidade de Campo dos Goytacazez, RJ 2004-2006.

encefálico e o outro por insuficiência renal, em paciente já em programa de diálise. Os demais (42\%) foram mantidos em evolução clínica, com registro de 11 óbitos (21,2\%) (fig. 3).

Ao todo foram realizadas 93 angioplastias coronarianas, abordadas 139 lesões (1,5 lesões por paciente), com implante de 121 stents, dos quais sete foram farmacológicos (1,3 stents por paciente). $\mathrm{O}$ uso do balão intra-aórtico foi muito baixo, tendo sido utilizado em apenas seis pacientes (3,8\%), o mesmo ocorrendo com o uso de inibidores da glicoproteína IIb/IIla, indicados apenas para dez (6,3\%) pacientes (tab. 3).

Houve insucesso na realização da angioplastia em quatro pacientes $(4,3 \%)$, oclusões subagudas em dois deles $(2,2 \%)$, sangramento importante no local de punção em outros dois $(2,2 \%)$, dissecção do TCE em um único paciente $(1,1 \%)$ e pseudo-aneurisma em somente um deles (1,1\%). Não houve óbitos durante a realização dos procedimentos. No entanto, ocorreram dois óbitos $(2,2 \%)$ na evolução hospitalar: um deles decorrente de possível ruptura cardíaca no terceiro dia de evolução após ATC de resgate com sucesso, e o outro, uma paciente triarterial, com insucesso na angioplastia, que evoluiu com disfunção ventricular grave e arritmia (tab. 4).

A letalidade global foi de 9,5\%, considerando os seguintes tratamentos: clínico, angioplastia coronária nas suas diversas formas e cirúrgico (fig. 4).

\section{Discussão}

A população estudada era idosa, porém com um número não desprezível de pacientes abaixo de 50 anos de idade. Em

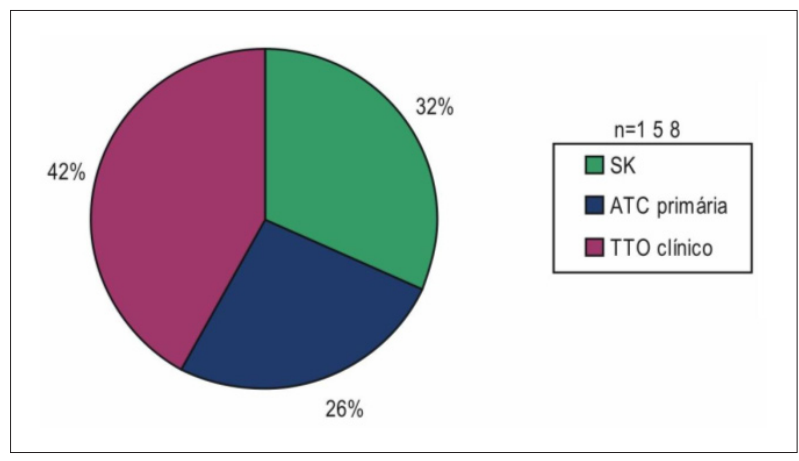

Fig. 2 - Tratamento inicial dos pacientes com IAM com SST na cidade de Campos dos Goytacazes, RJ 2004-2006.

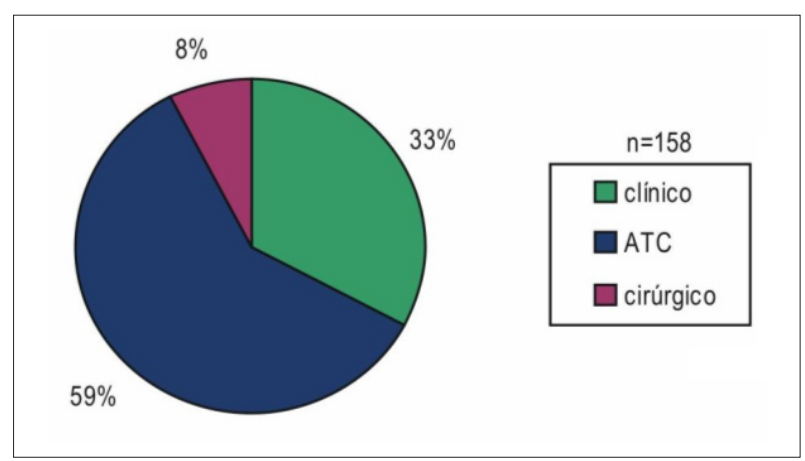

Fig. 3 - Tratamento definitivo dos pacientes com IAM com SST na cidade de Campos dos Goytacazes, RJ 2004-2006. 
Tabela 3 - Número de lesões abordadas por ATC, número e tipo de stents utilizados nos pacientes com IAM com SST na cidade de Campos dos Goytacazes, RJ, 2004-2006

\begin{tabular}{lcc}
\hline & $\mathbf{n}$ & $(\%)$ \\
\hline Número lesões dilatadas & 139 & 100 \\
\hline ATC com 1 stent & $69 / 93$ & 74,1 \\
\hline ATC com 2 stents & $20 / 93$ & 21,5 \\
\hline ATC com 3 stents & $4 / 93$ & 4,3 \\
\hline Total de stents utilizados & $121 / 93$ & 1,3 (média) \\
\hline Stents convencionais & 114 & \\
\hline Stents farmacológicos & 7 & 1,5 (média) \\
\hline Media de lesões dilatadas & $139 / 93$ & \\
\hline
\end{tabular}

Tabela 4 - Complicações relacionadas com as angioplastias coronarianas e evolução dos pacientes com IAM com SST na cidade de Campos dos Goytacazes, RJ, 2004-2006

\begin{tabular}{lcc}
\hline Complicação & $\mathrm{n}(\%)$ & Evolução \\
\hline Insucesso na recanalização & $4 / 93(4,3)$ & $\begin{array}{c}\text { Três com boa evolução e um } \\
\text { óbito por hipopotassemia }\end{array}$ \\
\hline Óbito hospitalar & $2 / 93(2,2)$ & $\begin{array}{c}\text { Um óbito por ruptura cardíaca 48 } \\
\text { horas após procedimento }\end{array}$ \\
\hline Sangramentos & $2 / 93(2,2)$ & Boa evolução \\
\hline Dissecção do tronco & $1 / 93(1,1)$ & $\begin{array}{c}\text { Cirurgia de revascularização } \\
\text { com bom resultado }\end{array}$ \\
\hline Pseudo aneurisma & $1 / 93(1,1)$ & Boa evolução \\
\hline Oclusão subaguda & $2 / 93(2,2)$ & Recanalização (boa evolução) \\
\hline
\end{tabular}

relação aos dados socioeconômicos e culturais dos pacientes, observamos que a renda familiar assemelha-se à da população geral da cidade e que $65,4 \%$ das famílias vivem com até cinco salários mínimos. Houve grande prevalência de pacientes com baixo nível de escolaridade, e aproximadamente $55 \%$ deles cursaram somente até a quarta série primária, com apenas $10 \%$ com nível superior, fatores que vem ganhando destaque em publicações recentes ${ }^{5}$.

A prevalência dos principais fatores de risco foi alta, exceto para o tabagismo, que pode ser explicada, talvez, pelo grande número de mulheres não-fumantes e de ex-fumantes entre os pacientes de grupos etários mais elevados. Na comparação da história prévia de doença coronariana entre os grupos de pacientes do nosso próprio material - IAM com SST vs. angina instável vs. IAM sem SST -, observamos que a história de DAC prévia era menos prevalente no primeiro deles, sendo o IAM a primeira manifestação da doença coronariana.

Considerando o registro da alta prevalência dos principais fatores de risco neste estudo, houve baixa utilização prévia de medicamentos, estando os pacientes insuficientemente tratados, visto que apenas $4,5 \%$ deles utilizavam os três medicamentos básicos associados, apesar de esses fármacos estarem disponíveis nas farmácias de toda a rede hospitalar. Esses pacientes também foram medicados de maneira inadequada no momento da alta hospitalar, quando apenas $57 \%$ deles foram orientados a fazer uso da associação de aspirina, betabloqueador e estatina.

Houve uma taxa elevada de estudos cinecoronariográficos $(93,7 \%)$, talvez em decorrência da relativa dificuldade na realização dos exames não-invasivos para estratificação dos pacientes, dados estes compatíveis com os de Santos e cols. ${ }^{6}$, que realizaram estudo cinecoronariográfico em $92,3 \%$ dos pacientes internados com IAM com SST. Com exceção do eletrocardiograma, realizado em todos os pacientes do nosso estudo, a maioria não-seriado, os demais exames, tais como ecocardiograma e cintilogafia miocárdica, foram utilizados muito aquém do esperado.

Também houve uma maior disponibilidade do serviço de hemodinâmica durante as 24 horas do dia, e a maior parte desses procedimentos foi realizada em hospitais de referência para onde eram encaminhados os pacientes, já com prévia indicação do estudo cinecoronariográfico.

A maior parte dos pacientes chegou ao hospital em tempo hábil para a realização de tratamento de reperfusão mecânica ou química, que, no entanto, não foi realizado em aproximadamente $35 \%$ deles, tendo apresentado taxas muito abaixo das expectativas. Quando realizado, predominou o tratamento com estreptoquinase endovenosa, seguida pela ATC primária. O tratamento definitivo usado com maior frequência foi a ATC, nas suas diversas formas (primária, resgate e eletiva), que foi feita em $59 \%$ dos pacientes, seguida pelo tratamento clínico, realizado em $33 \%$ dos pacientes, e o tratamento cirúrgico, em $8 \%$ deles. As taxas de letalidade foram baixas para ATC e altas para os tratamentos clínico e cirúrgico, no entanto tratava-se de uma amostra muito pequena cujos pacientes apresentavam maiores comorbidades.

No que se refere ao tipo de tratamento de reperfusão adotado, observamos que cerca de $40 \%$ dos pacientes submetidos a angioplastia primária tiveram um tempo portabalão de até 90 minutos, considerado bom, e ainda melhor, se levarmos em conta que esses procedimentos foram realizados na sua maioria em pacientes atendidos em hospitais gerais e encaminhados para um hospital de referência, estando aí incluído o retardo durante o transporte até este hospital ${ }^{7}$. Chama atenção ainda o fato de que apenas $10 \%$ dos pacientes chegaram ao pronto-atendimento após doze horas do início dos sintomas. Observamos também que o tipo de tratamento recebido apresentava variações conforme o dia da semana ${ }^{8}$ ou as equipes de plantão. Outro fato importante foi que, em algumas ocasiões, não havia leitos disponíveis nas unidades coronarianas dos hospitais da cidade, mesmo naqueles com serviço de hemodinâmica 24 horas por dia, o que impediu o tratamento adequado de uma parte dos pacientes.

No tocante à utilização dos stents, verificou-se predomínio dos convencionais, tendo sido usados os farmacológicos em apenas sete casos, já que não existem dados da literatura que comprovem seus benefícios na evolução tardia desses pacientes, embora possam reduzir o número de reinternações 9 . No total, foram abordadas 139 lesões em 93 pacientes $(1,5$ lesões/paciente) e utilizados 1,3 stents/paciente, número este bem próximo dos dados publicados pela Central Nacional de Intervenções Cardiovasculares ${ }^{10}$. 


\section{Artigo Original}

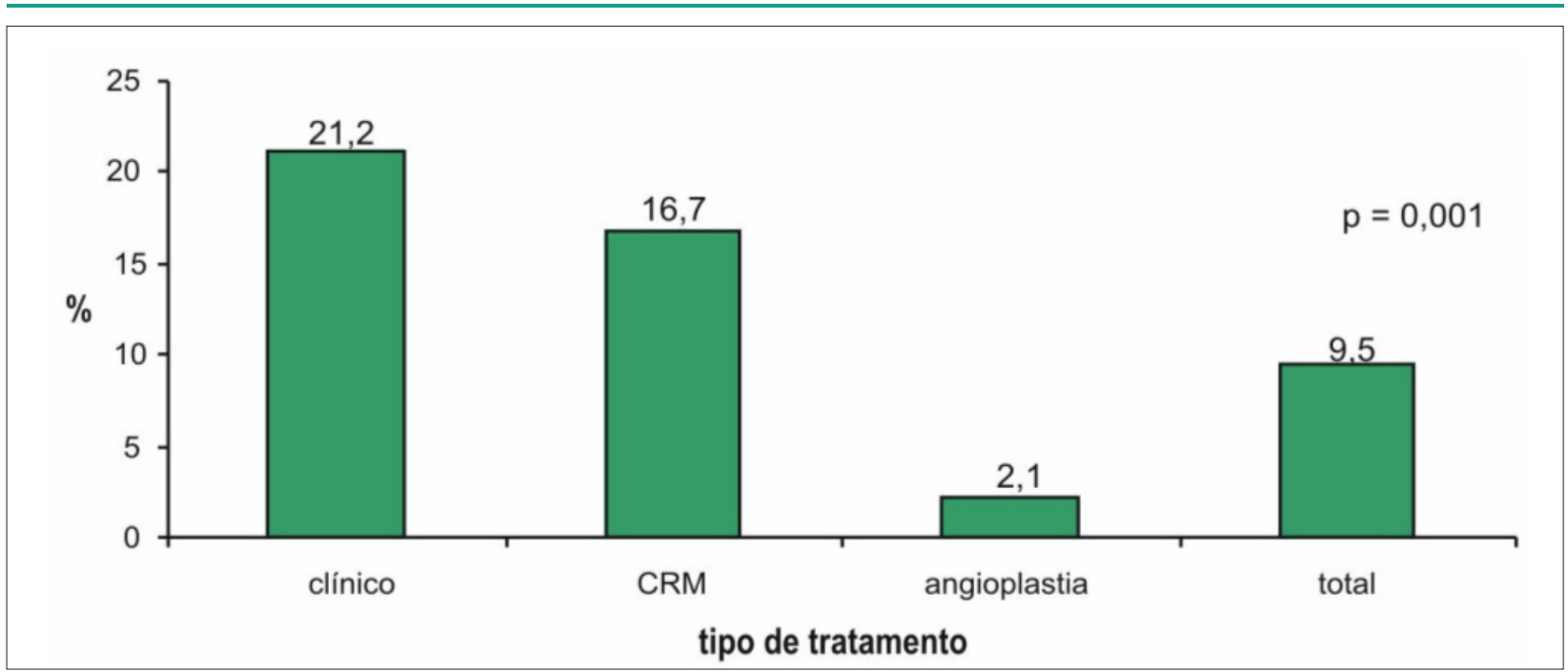

Fig. 4 - Óbito por tratamento definitivo dos pacientes com IAM com SST na cidade de Campo dos Goytacazes, RJ 2004-2006; CRM - cirurgia de revascularização miocárdica.

Na maioria das vezes, abordou-se apenas a artéria culpada, e em 18 lesões não se utilizaram stents. A letalidade dos pacientes submetidos a ATC esteve em torno de 2,2\%, percentual este considerado baixo, se comparado ao dos tratamentos clínico e cirúrgico, no entanto os pacientes submetidos a tratamento cirúrgico, com letalidade de 16,7\%, apresentaram maior número de comorbidades. Além disso, foi uma amostra muito pequena, da qual não podemos tirar conclusões. Quanto ao tratamento clínico, que também apresentou uma letalidade alta 11/53 (21,2\%), incluía o grupo de pacientes mais graves, com disfunções ventriculares, choque cardiogênico, lesões multiarteriais difusas e disfunções mitrais severas. Acreditamos que uma abordagem mais invasiva desse subgrupo poderia ter contribuído para um melhor resultado final ${ }^{11}$.

A baixa taxa de tratamento de reperfusão observada neste nosso estudo, embora relatada em vários trabalhos, não se justifica em uma cidade de porte médio, como é o caso de Campos, que possui dez hospitais situados no centro do município, além de uma rede de mais de cem postos médicos distribuídos pelo interior, e é dotada de serviço de transporte e comunicação, bem como de estradas com razoável estado de conservação e sem maiores problemas ligados ao trânsito, o que facilita alcançar os maiores hospitais em menos de 60 minutos, diminuindo assim o tempo porta-balão. De fato, nossos pacientes, de um modo geral, chegaram a tempo para reperfusão, porém não receberam o tratamento, o que reitera a necessidade de uma otimização dos serviços de emergência cardíaca, para que possamos atender melhor essa população de pacientes e, dessa forma, contribuir para a diminuição da morbidade e também da letalidade, que apresenta altas taxas não só na fase hospitalar, mas também na sua evolução após a alta ${ }^{12}$.

Concluímos que a mortalidade do IAM com SST pode ser reduzida, porém, para que seja alcançado esse objetivo, tornam-se necessárias estratégias eficazes de redução dos tempos de atendimento e de reperfusão, e que esta química ou mecânica - seja de fato disponibilizada para todos os pacientes com IAM ${ }^{13}$. Além disso, é importante que se viabilize para esses pacientes um suporte clínico adequado não só relacionado com as medicações associadas, mas também no que se refere a condutas que possam melhorar a sua evolução. Na verdade, todas essas condutas já estão bem estabelecidas, embora muitas vezes não realizadas, talvez por falta de equipes médicas bem treinadas e em número bastante para atender à demanda crescente nos setores de emergência cardiológicas dos hospitais em todo o país.

\section{Limitações}

O presente estudo apresenta limitações pelo fato de a amostra, embora representativa de uma população de cidade de porte médio, ter sido muito pequena e com acompanhamento por tempo limitado.

\section{Potencial Conflito de Interesses}

Declaro não haver conflito de interesses pertinentes.

\section{Fontes de Financiamento}

O presente estudo não teve fontes de financiamento externas.

\section{Vinculação Acadêmica}

Este artigo é parte de Dissertação de Mestrado de Jamil da Silva Soares pela Universidade Federal Fluminense - Hospital 


\section{Referências}

1. Beaglehole R. International trends in coronary heart disease mortality on incidence rates. J Cardiovasc Risk. 1999; 6: 63-8.

2. Murray CJL, Lopez AD. The global burden of disease: a comprehensive assessment of mortality and disability from disease, injuries and risk factors in 1990 and projected to 2020. Massachussets: Harvard University Press; 1996.

3. Thom T, Haase N, Rosamond W, Howard VJ, Rumsfeld J, Manolio T, et al. Heart disease and stroke statistics - 2006 update. A report from the American Heart Association Statistics Committee and Stroke Statistics Subcommittee. Circulation. 2006; 113: e85-e151.

4. Yusuf S, Mehta SR, Chrolavicius S, Afzal R, Pogue J, Granger CB, et al. The OASIS-6 trial group: effects of fondaparinux on mortality and reinfarction in patients with acute ST-segment elevation myocardial infarction. The OASIS-6 randomized trial. JAMA. 2006; 295: 1519-30.

5. Piegas LS, Avezum A, Pereira JC, Neto JM, Hoepfner C, Farran JA, et al. AFIRMAR Study Investigators. Risk factors for myocardial infarction in Brazil Am Heart J. 2003; 146: 331-8.

6. dos Santos ES, Minuzzo L, Pereira MP, Castillo MT, Palácio MA, Ramos RF, et al. Registro de síndrome coronariana aguda em um centro de emergências em cardiologia. Instituto Dante Pazzanese de Cardiologia. Arq Bras Cardiol. 2006; 87: 597-602.

7. Dalby M, Bouzamondo A, Lechat P, Montalescot G. Transfer for primary angioplasty versus immediate thrombolysis in acute myocardial infarction: a meta-analysis. Circulation. 2003;108: 1809-14.
8. Kostis WI, Demissie K, Marcella SW, Shao YH, Wilson AC, Morayra AE, et al. for the Myocardial Infarction Data Acquisition System (MIDAS 10) study group. Weekend versus weekday admission and mortality from myocardial infarction. N Engl J Med. 2007; 356: 1099-109.

9. Kastrati A, Mehilli J, Pache J, Kaiser C, Valgimigli M, Kelbaek H, et al. Analysis of 14 trials comparing sirolimus-eluting stens with bare-metal stents. N Engl J Med. 2007; 356: 1030-9.

10. Sociedade Brasileira de Cardiologia. Sociedade Brasileira de Hemodinâmica e Cardiologia Intervencionista. Central Nacional de Intervenções Cardiovasculares (CENIC). São Paulo; 2004.

11. Sanborn TA, Sleeper La, Bates ER, Jacobs AK, Boland J, French JK, et al. Impact of thrombolysis intra-aortic balloon pump contrapulsation, and their combination in cardiogenic shock complicating acute myocardial infarction: a report from the SHOCK trial Registry. Should we emergently revascularize Occluded Coronaries for Cardiogenic Shock? J Am Coll Cardiol. 2000; 36 (Suppl A): 1123-9.

12. Solomon SD, Zelenkofske S, Mc Murray JJV, Finn PV, Velazquez E, Ertl G, et al. for the Valsartan in Acute Myocardial Infarction Trial (VALIANT) Investigators. Sudden death in patients with myocardial infarction and left ventricular dysfunction, heart failure, or both. N Engl J Med. 2005; 352: 2581-8.

13 Bradley EH, Herrin J, Wang Y, Barton BA, Webster TR, Mattera JA, et al. Strategies for reducing the door-to-balloon time in acute myocardial infarction. N Engl J Med. 2006; 355: 2308-20. 